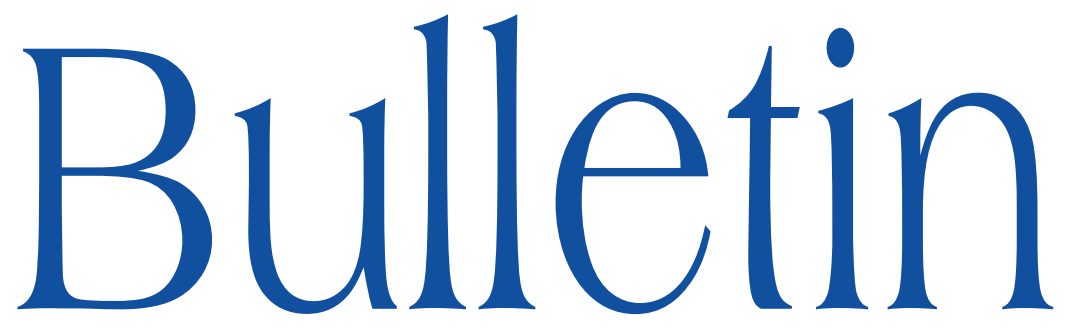

de la SOCIÉTÉ MATHÉMATIQUE DE FRANCE

\title{
ABOUT THE GENERATING FUNCTION OF A LEFT BOUNDED INTEGER-VALUED RANDOM VARIABLE
}

Charles Delorme \& Jean-Marc Rinkel

Tome 136

Fascicule 4

2008 


\title{
ABOUT THE GENERATING FUNCTION OF A LEFT BOUNDED INTEGER-VALUED RANDOM VARIABLE
}

\author{
By Charles Delorme \& JeAn-Marc Rinkel
}

\begin{abstract}
We give a relation between the sign of the mean of an integer-valued, left bounded, random variable $X$ and the number of zeros of $1-\Phi(z)$ inside the unit disk, where $\Phi$ is the generating function of $X$, under some mild conditions

RÉsumé (Fonction génératrice d'une variable aléatoire à valeurs entières minorées)

Nous donnons une relation entre le signe de l'espérance d'une variable aléatoire à valeurs entières minorées et le nombre des zéros de $1-\Phi(z)$ dans le disque unité où $\Phi$ est la fonction génératrice de $X$ et ce sous des conditions peu exigeantes.
\end{abstract}

\section{Introduction}

We consider an integer-valued, left bounded, random variable $X$. Its generating function $\Phi$ is $z \mapsto \sum_{k \in \mathbb{Z}} c_{k} z^{k}$, with $c_{k}=\mathbf{P}(X=k)$. If $X$ is left bounded, with $\inf (X)=-p$, we have $\Phi(z)=\sum_{k \geq-p} c_{k} z^{k}$. The object of this paper is the evaluation of the number of zeros inside the unit disc of the function $z \mapsto 1-\Phi(z)$. A motivation for counting these zeros is the exact and asymptotical estimation of the potentials related to random walks on an interval $[0, N]$ defined by $S_{n}=S_{0}+X_{1}+\cdots+X_{n}$ where $X_{i}$ 's are iid with integer left bounded

Texte reçu le 2 juillet 2007, révisé et accepté le 7 mai 2008

Charles Delorme, LRI Université Paris Sud, 91405 Orsay Cedex •

E-mail : Charles.Delorme@lri.fr

Jean-Marc Rinkel, Département de mathématiques, Université Paris Sud, 91405 Orsay

Cedex • E-mail : jean-marc.rinkel@math.u-psud.fr

2000 Mathematics Subject Classification. - 60G50, 47B35, 30Cxx.

Key words and phrases. - Random walk, random variable, generating function. 
distribution and $S_{0}$ is an integer valued random variable distributed on the interval $[0, N]$. These potentials are given by the entries of the inverse of Toeplitz matrices (see [3]). But the computation of the inverse uses the factorisation of the function $\theta \mapsto 1-\Phi\left(e^{i \theta}\right)$, called the symbol of the Toeplitz operator. One can read [2] for a detailed account. A theorem summarises the results obtained in this paper.

THEOREM 1. - Let $X$ be a integer valued left bounded random variable, $z \mapsto$ $\Phi(z)=\sum_{k \geq-p}^{\infty} c_{k} z^{k}$ its generating function, where $p=-\inf (X)>0$. We denote by $\rho$ the convergence radius of the series $\sum_{k \geq-p} c_{k} z^{k}$, $\zeta$ the number of zeros (taking multiplicities into account) inside the unit disc of $z \mapsto 1-\Phi(z), d$ the GCD of the $k$ 's such that $c_{k}>0$, in other words $d$ is the period of $X$. Then

$$
\zeta=\left\{\begin{array}{l}
-\inf (X) \text { if } E(X) \in(0, \infty] \\
-d-\inf (X) \text { if }\left\{\begin{array}{l}
E(X)<0 \text { or } \\
E(X)=0 \text { and } X \text { has a variance. }
\end{array}\right.
\end{array}\right.
$$

If $\rho=1$ and $E(X)=0$, then $\zeta \leq-d-\inf (X)$.

If $p \leq 0$, then obviously $1-\Phi$ has no zero inside the unit disk (except for the very uninteresting case where $c_{0}=1$ ), because the absolute value of a sum is at most the sum of the absolute value of its terms.

The random variable $X$ is aperiodic precisely when this $d=1$.

ConjeCture 1. - With the hypotheses and notations of Theorem 1, we have $\zeta=-d-\inf (X)$ even if $E(X)=0$.

In order to support the conjecture let us notice that for $E(X)=0$, some other classes of functions give again $p-d$ as the number of zeros. For example, if $p=1$ and $d=1$ (obviously) or $p=2$ : indeed there is at least one zero on the interval $(-1,0)$ when $p$ is even since $\Phi(-1)<1$ (see lemma 1 ) and $\left.\lim _{x \rightarrow 0^{-}} \Phi(x)=+\infty\right)$.

REMARK 1. - It suffices to prove the theorem (or the conjecture) in the case $d=1$ to obtain the general one. In fact, this is obtained by noticing that $\Phi(z)=\phi\left(z^{d}\right)$ with $\phi$ the generating function of the integer-valued and aperiodic random variable $X / d$. Denoting by $\zeta_{1}$ the number of zeros (with multiplicities) inside the unit disc of $1-\phi$, we have then $\zeta=d \zeta_{1}$. We deduce that $\zeta=p=$ $-\inf (X)$ when $\zeta_{1}=p / d=-\inf (X / d)$ and $\zeta=-d+p=-d-\inf (X)$ when $\zeta_{1}=-1+p / d=-1-\inf (X / d)$.

From now on, we will consider only the case $d=1$.

TOME $136-2008-\mathrm{N}^{\circ} 4$ 


\section{Some lemmas}

We will use two well-known propositions

LEMMA 1 (Generalized triangle inequality). - Let $a_{i}, 0 \leq i$ be an absolutely convergent series of complex numbers. Then $\left|\sum_{i=0}^{\infty} a_{i}\right| \leq \sum_{i=0}^{\infty}\left|a_{i}\right|$. Moreover, the equality occurs only if the non-null terms have the same argument. Of course, this common argument is also the argument of the sum itself.

LEMma 2 (Rouché's theorem [1, p. 13]). - Let $D$ a domain in the complex plane, let $C$ be a simple closed curve and let $f, g$ be two functions, meromorphic on $D$, having no pole on $C$, such that $|g(z)|<|f(z)|$ for all $z$ of $C$. Then $f+g$ and $f$ have the same total number of zeros and poles, counted with multiplicities, inside $C$.

LEMMA 3. - Let $p$ be a positive integer and $c_{k}, k \geq p$ be a sequence of nonnegative reals, such that

- $c_{-p}>0$,

- $\sum_{k \geq-p} c_{k}=1$,

- the GCD of the $k$ 's such that $c_{k}>0$ is 1 .

Then the series $\Phi(z)=\sum_{k \geq-p} c_{k} z^{k}$ converges on the punctured disk $0<|z| \leq$ 1 , its sum is continuous on that disk, and meromorphic on the open disk $|z|<1$. The convergence of the series to its sum is uniform. The only point on the circle $|z|=1$ where $\Phi(z)=1$ is 1 .

Proof. - The remainder of the series is $\sum_{k \geq N} c_{k} z^{k}$ for $N \geq 0$ is at most $\sum_{k>N} c_{k}$ since $\left|z^{k}\right| \leq 1$ and $c_{k} \geq 0$. Since the series converges at the point $z=1$, we have proven the uniform convergence. The continuity of $\Phi$ and its being meromorphic are classical consequences of this uniform convergence.

Let us consider the set $S$ of integers such that $c_{k}>0$. The equality $\sum_{S} c_{k} z^{k}=1=\sum_{S} c_{k}$ with $|z|=1$ and $c_{k} \geq 0$ imposes that $z^{k}=1$ for all $k$ 's in $S$, due to the generalized triangle inequality. Since the GCD of these $k$ 's is 1 , there is a finite part $T$ of $S$ and integers $\beta_{k}, k \in T$ such that $\sum_{k \in T} \beta_{k} k=1$ Then

$$
z=z^{\Sigma_{k \in T} \beta_{k} k}=\prod_{k \in T}\left(z^{k}\right)^{\beta_{k}}=1
$$

and 1 is the only point on the unit circle where $\Phi=1$.

LEMMA 4. - The restriction of $\Phi(z)=\sum_{k \geq-p} c_{k} z^{k}$ to the real interval $(0,1]$ either takes value 1 at point 1 only or takes value 1 at an other point $r$ inside the interval $(0,1)$. In the first case, $X$ has a mean $E(X)=\sum_{k \geq-p} k c_{k}$ and $E(X) \leq 0$. In the second case it has a positive mean or the series $\sum_{k \geq-p} k c_{k}$ diverges to $+\infty$, and $\Phi(x)<1$ on the interval $(r, 1)$ 
Proof. - This results from the observation that the restriction of $\Phi$ is continuous, strictly convex, and $\Phi(x) \rightarrow \infty$ when $x \rightarrow 0^{+}$.

\section{The case with no mean value or positive mean value}

In this case, let $r$ be the point in $(0,1)$ where $\Phi(r)=1$. For each radius $s$ such that $r<s<1$, we see that on the circle centered at 0 with radius $s$ we have $|\Phi(z)| \leq \Phi(s)<1$. This circle is inside the domain where $\Phi$ is meromorphic. By Rouché's theorem, we can assert that the number of zeros and poles counted with their order inside the circle of radius $s$ is the same for the function 1 and the function $1-\Phi$. This common number is of course 0 , since the function 1 has neither zeros nor poles! Since $1-\Phi$ has just a pole of order $p$, it has zeros whose orders sum up to $p$ inside the disk of radius $s$. Since the open unit disk is the union of the open disks of radiuses $s<1$, it contains $p$ zeros counted with their order.

We moreover see that $r$ is the zero of $1-\Phi$ with largest absolute value inside the unit disk, and it is the only zero of absolute value $r$, again an application of the generalized triangle inequality. It is simple, since $\Phi^{\prime}(r)<0$.

\section{The case of negative mean value and convergence radius $>1$}

A proof in the same vein allows also to estimate the number of zeros when the convergence radius is $>1$ and the mean value $<0$. Since the derivative of $\Phi$ at 1 is $<0$, there is a real $r$, smaller than the convergence radius, with $1<r$ such that $\Phi(x)<1$ on the interval $(1, r]$. With the same trick as in the former section, the circles of radius $x$ with $1<x<r$ do not contain zeros of $1-\Phi$, because $|\Phi(z)| \leq \Phi(x)<1$ for $|z|=x$. Thus the disk of radius $r$ contains only zeros of $1-\Phi$ of absolute value $\leq 1$. According to Rouché's theorem, the number of zeros and poles (counted with their order) of 1 and $1-\Phi$ inside the disk of radius $x$ coincide. This number is 0 . Because of the pole of order $p$ at 0 , there are $p$ zeros (counted with their order) inside the disk of radius $r$, one of them is the simple zero at 1 , and it is the only one with absolute value 1 .

Thus the open disk of radius 1 contains $p-1$ zeros of $1-\Phi$ (counted with their order).

\section{The case of mean value $\leq 0$ and convergence radius 1 : an inequality}

Since the function $1-\Phi$ is meromorphic on the open unit disk, its zeros inside the open disk are isolated. However they may accumulate towards the unit circle. Since the function is continuous on the punctured disk, the only possible limit of a sequence of zeros of $1-\Phi$ is the point 1 .

TOME $136-2008-\mathrm{N}^{\mathrm{O}} 4$ 
Consider a crown $r \leq|z| \leq s$ with $0<r<s<1$. Since this crown is compact, it can contain only a finite number of zeros of $1-\Phi$. Thus there is a radius $t$ between $r$ and $s$ such that the circle centered at 0 with radius $t$ contains no zero of $1-\Phi$. By continuity of $1-\Phi$ and compacity of the circle, the absolute value of $1-\Phi$ on this circle has a positive minimum $m$.

Now, since the convergence of the series is uniform, there is an integer $N$ such that the remainder $R_{N}=\sum_{k>N} c_{k} z^{k}$ satisfies $2\left|R_{N}\right|<m$ on the unit disk. Then the functions $1-\Phi$ and $1-\Phi_{N}=1-\left(\Phi-R_{N}+R_{N}(1)\right)$ have the same number of zeros and poles inside the disk of radius $t$ (according to Rouché's theorem). We notice then that $\Phi_{N}$ is for $N>0$ the generating function of a random variable $X_{N}$ with distribution

- $P\left(X_{N}=0\right)=c_{0}+R_{N}(1)$,

- $P\left(X_{N}=k\right)=0$ if $k>N$,

- $P\left(X_{N}=k\right)=c_{k}$ if $(-p \leq k \leq N) \wedge(k \neq 0)$,

that has a negative mean. Indeed, $\sum_{-p \leq k \leq N} k c_{k} \leq \sum_{k \geq-p} k c_{k} \leq 0$.

Both inequalities are equalities only if $X$ has mean value 0 and $X \leq N$. But the convergence radius is then infinite. This case will be dealt with in section 7 .

Now $\Phi_{N}$ is a Laurent polynomial, the result of section 4 can be applied to it. The disk of radius $t$ contains at most $p-1$ zeros of $1-\Phi_{N}$ (some of the zeros of $1-\Phi_{N}$ in the unit disk could have absolute value larger than $t$ ) and also at most $p-1$ zeros of $1-\Phi$, since both have a pole of order $p$ at the origin.

Since the punctured unit open disk is the union of such disks of radius $t$ we can conclude that it contains at most $p-1$ zeros of $1-\Phi$ (counted with their order).

We have just proven

Proposition 1. - Let $X$ be a random variable, aperiodic, integer-valued and left-bounded, say $\inf (X)=-p$, such that $E(X) \leq 0$ and $\Phi$ has convergence radius 1. Then $\Phi-1$ has at most $p-1$ zeros (counted with multiplicity) inside the unit disk.

To prove that the number of zeros is $p-1$, we have to prove that no sequence of zeros of the functions $\Phi_{N}-1$ inside the unit disk tends to 1 . This can be done in some cases, as explained below (sections 6 and 7) 


\section{The case of negative mean value}

Instead of the series of monomials $c_{k} z^{k}$ with sum $\Phi$, we consider the sum of polynomials $c_{k}\left(z^{k}-1\right) /(z-1)$, whose value at 1 is $c_{k} k$. The series converges at 1 to $\Phi^{\prime}(1)=E(X)$. Moreover, the absolute value of $\left(z^{k}-1\right) /(z-1)=$ $z^{k-1}+z^{k-2}+\cdots+z+1$ on the unit closed disk is at most $k$ for $k>0$. Thus the convergence of the series towards its sum $\Psi(z)=(\Phi-1) /(z-1)$ for $z \neq 1$ (resp. $\left.\Psi(1)=\Phi^{\prime}(1)\right)$ is uniform on the unit closed disk. The sum is thus continuous and does not vanish on the unit circle, and therefore on a crown $r \leq z \leq 1$ with $r$ close enough to 1 . Using the minimum of $|\Psi(z)|$ and the uniform convergence, we see that $\Psi_{N}=\frac{\Phi_{N}-1}{z-1}$ has no zero in the crown when $N$ is large.

Thus $\Phi-1$ has $p-1$ zeros in the unit open disk.

\section{The case of null mean}

If the set $S$ of indices $k$ such that $c_{k}>0$ is infinite, we can apply the method of section 5 , and come to the conclusion that $1-\Phi$ has at most $p-1$ zeros inside the unit disk.

7.1. Right bounded variables. - If the set is finite, with maximum $M$ (certainly positive, because of the necessary compensation of the term $-p c_{-p}$ ), we can introduce the variable $X_{\epsilon}$ whose generating function $\Phi_{\epsilon}$ is $\Phi+\epsilon\left(1-z^{M}\right)$, where $\epsilon$ is a small positive real. This $X_{\epsilon}$ has a negative mean. Having chosen a radius $t \in(0,1)$ such that the circle of radius $t$ encircles the (clearly finite) set of zeros of $\Phi$ inside the open unit disk, we can choose $\epsilon$ in order to have $\left|\epsilon\left(1-z^{M}\right)\right|<m$, where $m$ is the minimum of $1-\Phi$ on the circle of radius $t$. Then $1-\Phi$ and $1-\Phi_{\epsilon}$ have the same number of zeros inside the disk of radius $t$, that is at most $p-1$.

The random variable $-X$, whose mean is also null, has the generating function $\Phi(1 / z)$. The function $1-\Phi(1 / z)$ has at most $M-1$ zeros inside the unit disk. The inverses of these zeros are zeros of $1-\Phi$ with the same order outside the unit disk. Thus the polynomial $z^{p}(1-\Phi(z))$ of degree $p+M$ has $p+M$ zeros counted with their order, at most $p-1$ inside the unit disk, at most $M-1$ outside the unit disk, and at most two at point 1 , since $\Phi$ is strictly convex. Therefore the three quantities must be equal to their upper bounds.

In particular, $z^{p}(1-\Phi(z))$ has $p-1$ zeros inside the open disk of radius 1 , as well as $1-\Phi$. 
7.2. Variables with a variance. - This equality remains valid when $X$ admits a variance given from the generating function by $V(X)=\Phi^{\prime \prime}(1)+\Phi^{\prime}(1)-\Phi^{\prime}(1)^{2}=$ $\Phi^{\prime \prime}(1)$, that is clearly $>0$. This happens in particular when the convergence radius is $>1$.

We consider then a series designed to converge to $z^{p}(\Phi-1) /(z-1)^{2}$, namely the one with terms $c_{k} A_{k}(z) / p$, where $A_{k}=\left(p z^{k+p}+k-(p+k) z^{p}\right) /(1-z)^{2}$ is a polynomial whose value at 1 is $k(p+k)$, its coefficients are positive for $k \geq 1$ namely $A_{k}=\sum_{s=0}^{p-1} k(s+1) z^{s}+\sum_{t=1}^{k-1} p t z^{p+k-1-t}$. For example, with $p=3$ and $k=2$, it is $2+4 z+6 z^{2}+3 z^{3}$. Therefore $\left|A_{k}(z)\right| \leq A_{k}(1)$ if $|z| \leq 1$ and $k \geq 1$. Thus the series has uniform convergence on the closed unit disk. The partial sum $\sum_{-p \leq k \leq N} c_{k} A_{k} z^{k} / p$, when $N$ is large enough, corresponds to the similar construction applied to a random variable $Y_{N}$ with $\inf \left(Y_{N}\right)=-p$, $\sup \left(Y_{N}\right) \leq N$ and $E\left(Y_{N}\right)=0$, whose generating function has $p-1$ zeros inside the unit disk.

The distribution of $Y_{N}$ is given by the rules

- $\mathbf{P}\left(Y_{N}=-p\right)=c_{-p}-\alpha_{N}$,

- $\mathbf{P}\left(Y_{N}=0\right)=c_{0}+\alpha_{N}+\beta_{N}$,

- $\mathbf{P}\left(Y_{N}=k\right)=0$ if $k>N$,

- $\mathbf{P}\left(Y_{N}=k\right)=c_{k}$ if $(-p<k \leq N) \wedge(k \neq 0)$,

where $\alpha_{N}$ is the remainder $\sum_{t>N} t c_{t} / p$ and $\beta_{N}$ is the remainder $\sum_{k>N} c_{t}$. This makes sense if $N$ is large enough to ensure $\alpha_{N}<c_{-p}$.

Once again, by continuity, the sum of the series has no zero on a crown $r \leq|z| \leq 1$ with $r$ close enough to 1 , and uniform convergence provides a $N$ such that the generating functions of $X$ and $Y_{N}$ have the same number of zeros on the unit disk, namely $p-1$ according to subsection 7.1 since $Y_{N}$ is right bounded.

In that case again, we have proven that $\Phi-1$ has $p-1$ zeros inside the unit disk.

However the case where $E(X)=0$ and $X$ has no variance remains problematic.

Some other classes of function give again $p-1$ as the number of zeros. For example, if $p=1$ (obviously) or $p=2$ (there is at least one zero on the interval $(-1,0)$ when $p$ is even since $\Phi(-1)<1$ and $\left.\lim _{x \rightarrow 0^{-}} \Phi(x)=+\infty\right)$.

\section{Another approach}

Let us sketch another way to evaluate the number of zeros of $\Phi-1$

We have seen that $|\Phi(z)| \leq 1$ on the unit circle. On the other hand, near the origin, $|\Phi(z)|$ is large. Thus there is a closed connected region surrounding the origin where $|\Phi| \geq 1$; on its border $C$, we have $|\Phi|=1$. 
If $E(X) \leq 0$, this border $C$ attains the point 1 and does not meet the unit circle elsewhere. If $E(X)>0$, then $C$ remains in the closed disk centered at the origin with radius $r$, where $0<r<1$ and $\Phi(r)=1$.

On the other hand, the part of $\mathbb{C}$ where $\Phi(z)$ is positive real is a curve, that has $p$ branches starting at the origin. This curve may have some crossings. The points where it meets $C$, are $p$ zeros of $\Phi-1$ (counted with multiplicities). If $E(X)>0$, all $p$ intersections of $C$ and the curves where $\Phi>0$ are inside the unit disk, if $E(X) \leq 0$, one of them is the point 1 . There are $p-1$ left inside provided the zero at 1 is simple.

If $\Phi$ admits around the point 1 a meromorphic continuation in a Riemann surface, say $z=Z(t)$ and $\Phi(z)=F(t)$ are meromorphic in a neighborhood of 0 , with $Z(0)=F(0)=1$ and $(F(t)-1) /(Z(t)-1)^{\alpha} \rightarrow c \neq 0$, in other words $\Phi-1$ has a zero in $z$ of order $\alpha$ at 1 , with $1<\alpha<2$, the curves $\Phi>0$ meet at 1 with angles $\pi / \alpha>\pi / 2$, an thus only one of them (namely the one with $z$ real between 0 and 1) can meet the point 1 from inside the unit disk. In this case, the number of zeros is $p-1$. Something similar can be done with exponentials, and $t$ going to $-\infty$.

A wider class of functions can be dealt with that way.

Let us give examples, where this idea can be applied, and another one where it is of no use.

8.1. An algebraic example. - The function $k(z)=4(1-z)^{5 / 4}-4+5 z=$ $\sum_{k=0}^{\infty} \frac{5 z^{k+2} \prod_{\ell=0}^{k-1}(4 \ell+3)}{4^{k+1}(k+2) !}$ constitutes the generating function of a random variable with mean 5 , minimum 2 and no variance. Thus $k(z) / z^{5}$ is the generating function of a random variable with mean 0 and no variance, and minimum -3 . As expected, the function $k(z) / z^{5}-1$ has exactly two zeros inside the unit disk: one is approximately $-0.4419+0.693 i$ and the other is its conjugate.

8.2. An example with logarithm. - The function $z \mapsto\left(1+9 z^{2}-6 z-6(1-\right.$ $\left.z)^{2} \ln (1-z)\right) /\left(4 z^{3}\right)$, whose series is $z^{-3} / 4+\sum_{n=0}^{\infty} \frac{3 z^{n}}{(n+1)(n+2)(n+3)}$ is the generating function of a random variable with mean 0 and minimum -3 , and no variance. As expected, the function has exactly two zeros inside the unit disk: one is approximately $-0.4052+0.6488 i$ and the other is its conjugate.

8.3. A transcendant example. - The function $T(z)=\frac{1}{3 z^{3}}+\frac{1}{2} \sum_{k=0}^{\infty} z^{2^{k}} / 4^{k}$ is the generating function of a variable with null mean and minimum -3 . Its derivatives satisfy $z T^{\prime}(z)+z^{2} T^{\prime \prime}(z)=3 z^{-3}+\sum_{k=0}^{\infty} z^{2^{k}}$ and the function $\sum_{k=0}^{\infty} z^{2^{k}}$ has a set of singularities dense on the unit circle. It falls in the hereto undecided case. However $T-1$ has two zeros inside the unit disk, one is close to $-0.35808+0.54023 i$ and the other is its conjugate. In this example also the conjecture is validated.

TOME $136-2008-\mathrm{N}^{\mathrm{O}} 4$ 


\section{Conclusion}

We conjecture that the number $\zeta$ of zeros (with multiplicities) of $\Phi-1$ inside the disk is $p-1$ if $X$ is aperiodic and $E=0$.

We have validated this conjecture except for some cases where $p>2$ and $E(X)=0$ and $X$ has no variance. In these cases, only the inequality $\zeta \leq p-1$ has been proven.

\section{BIBLIOGRAPHY}

[1] A. F. Beardon - A primer on Riemann surfaces, London Mathematical Society Lecture Note Series, vol. 78, Cambridge University Press, 1984.

[2] C. Delorme \& J.-M. Rinkel - "Application of the Toeplitz matrices to the asymptotic estimation of the potentials for random walks on the non negative integers with a left bounded generator", Prépublication de la faculté des sciences d'Orsay, 2007.

[3] F. Spitzer - Principles of random walk, 2nd ed., Springer Verlag, 2001. 